\title{
Determinantes del nivel de catalán de los inmigrantes en Cataluña: un análisis de sección cruzada a nivel comarcal*
}

\author{
Javier Ortega \\ Université de Toulouse (GREMAQ, IDEI), CEP (LSE), CEPR, FEDEA e IZA
}

\begin{abstract}
Resumen
Presentamos un análisis de sección cruzada a nivel comarcal para estudiar los determinantes del conocimiento activo oral del catalán de los residentes en Cataluña nacidos fuera de esta comunidad autónoma (los «inmigrantes»). El conocimiento del catalán de los inmigrantes es mayor en las zonas pirenaicas o agrícolas, y menor en el área metropolitana de Barcelona. El resto de los determinantes es diferente para diferentes grupos de inmigrantes. En su conjunto, los inmigrantes tienen un mayor conocimiento del catalán en aquellas comarcas en las que el conocimiento del catalán de los nacidos en Cataluña es mayor. Por su parte, el conocimiento del catalán de los inmigrantes nacidos en España es mayor en las comarcas en las que constituyen una fracción pequeña de los residentes. Por último, los inmigrantes nacidos fuera de España tienen un mayor conocimiento del catalán en las comarcas con mayor voto a partidos nacionalistas catalanes y en las comarcas de menor densidad, así como cuando su nivel de estudios es más alto.
\end{abstract}

Palabras clave: lenguas, inmigración, catalán

Clasificación JEL: F22, J15

\begin{abstract}
We present a cross-section analysis at the local level to study the determinants of the oral knowledge of Catalan of residents in Catalonia who were born outside this community (the «immigrants»). Immigrants' knowledge of Catalan is higher in the Pyrenean or agricultural areas and lower in the metropolitan area of Barcelona. The rest of the determinants differ across groups of immigrants. As a whole, immigrants have a higher level of Catalan knowledge in those areas where Catalan knowledge of people born in Catalonia is higher. On the other hand, Catalan knowledge of immigrants that were born in Spain is higher in those areas where they constitute a small fraction of residents. Finally, immigrants who were born out of Spain have a higher Catalan knowledge in areas with higher voting rates for Catalan nationalists and in those areas with lower density, as well as when their educational level is higher.
\end{abstract}

Keywords: languages, migration, Catalan

JEL Classification: F22, J15

*Agradezco los comentarios de Barbara Petrongolo y Maia Güell durante la elaboración de este artículo, así como las eficaces respuestas del IDESCAT a mis preguntas sobre los datos. 


\section{Introducción}

Casi treinta años después de la aprobación de la Constitución, el debate lingüístico sobre el uso del catalán y el castellano en Cataluña sigue abierto. Un elemento de este debate es el del nivel de conocimiento del catalán por parte de los residentes en Cataluña nacidos en el resto de España, así como, más recientemente, el conocimiento del catalán y del castellano de los residentes en Cataluña nacidos fuera de España. Para simplificar la terminología, nos referiremos a estos dos grupos como a los «inmigrantes» en Cataluña.

Pese a que Cataluña es una región con altos niveles de bilingüismo catalán-castellano tanto a nivel espacial como individual, la disponibilidad de datos sobre el conocimiento del catalán y del castellano es muy diferente. En efecto, los Censos Generales de Población y Vivienda incluyen desde 1986 en el cuestionario de hogar para Cataluña un apartado adicional sobre el conocimiento del catalán de los residentes en Cataluña. Dado que el Censo incluye una pregunta sobre el lugar de nacimiento de los censados, se conoce el nivel de catalán de los nacidos en Cataluña y de los inmigrantes. En cambio, los únicos datos disponibles sobre el conocimiento y uso del castellano en Cataluña (Estadística d'Usos Lingüístics a Catalunya, 2003) corresponden a una muestra realizada en 2003 a partir de 7257 entrevistas telefónicas. Aunque esta muestra incluye un mayor número de preguntas que el Censo lingüístico respecto al conocimiento y uso del castellano y el catalán, se dispone sólo de información agregada para cada uno de los siete «ámbitos territoriales» (reagrupamientos de comarcas) de Cataluña y no se dispone de información según el lugar de nacimiento de los individuos. Debido a esta limitación en la disponibilidad de datos para le castellano, nos centraremos en el estudio del conocimiento del catalán de los residentes en Cataluña. Sin embargo, dada la importancia del castellano en Cataluña, la interpretación de algunos de los resultados obtenidos para el catalán se basará en la información disponible sobre el uso del castellano.

Presentamos una serie de regresiones de sección cruzada a nivel de las 41 comarcas catalanas para estudiar el nivel de catalán de los inmigrantes. Nuestra variable endógena es la proporción de inmigrantes en cada comarca que declara saber hablar catalán. Escogemos este criterio de capacidad de utilización oral del catalán porque es un criterio intermedio respecto al de comprensión del catalán (de fácil cumplimiento debido a la proximidad lingüística del castellano y el catalán) y el de capacidad de escritura o lectura (de más difícil cumplimiento debido a las bolsas de analfabetismo y a la importante proporción de nacidos en Cataluña que no han aprendido a escribir el catalán pese a que lo hablan habitualmente).

En la lista de determinantes potenciales del nivel de catalán de los inmigrantes a nivel comarcal, incluimos en primer lugar la proporción de nacidos en Cataluña que declara hablar catalán y la proporción de inmigrantes. Estas dos variables pueden interpretarse como determinantes económicos. En efecto, si el aprendizaje del catalán es costoso (en términos monetarios o de tiempo) y el uso de la misma lengua es necesario para producir e intercambiar con otros individuos, un mayor conocimiento 
del catalán entre los nacidos en Cataluña hace más rentable el aprendizaje del catalán. Simétricamente, dado que la mayor parte de los inmigrantes son castellanoparlantes, una menor proporción de inmigrantes podría hacer también más rentable el aprendizaje del catalán.

En segundo lugar, dado que los partidos nacionalistas catalanes han puesto el énfasis en la defensa y desarrollo del catalán como elemento de una «identidad nacional» catalana, y que el aprendizaje del catalán podría tener una componente ideológica, introducimos también como variable explicativa el voto a los partidos nacionalistas catalanes (medido aquí como el voto comarcal a Convergència i Unió y Esquerra Republicana de Catalunya) como variable explicativa.

Las otras variables consideradas hacen referencia a las características económicas o demográficas de las comarcas, a la composición lingüística de los inmigrantes o a su nivel de estudios.

Estudiamos primero los determinantes del conocimiento del catalán para el conjunto de los inmigrantes en 2001. Mostramos que la proporción de nacidos en Cataluña que habla catalán en la comarca tiene un impacto positivo sobre el nivel de catalán de los inmigrantes, mientras que la proporción de inmigrantes en la comarca tiene un impacto negativo sobre esta misma variable. Al estudiar el peso relativo de estos dos factores económicos, obtenemos que el primero (correspondiente a la «fuerza de atracción del catalán») es más significativo estadísticamente que el segundo. Por su parte, el porcentaje de votos a los partidos nacionalistas catalanes tiene un impacto positivo y significativo sólo cuando no se incluyen las dos variables anteriores, lo cual parece indicar una menor importancia de los factores ideológicos. Cuando se incluye el nivel de catalán de los nacidos en Cataluña, un mayor porcentaje de votos a partidos nacionalistas tiene incluso un impacto negativo (no siempre significativo) sobre el conocimiento del catalán de los inmigrantes. En cuanto a la composición de la inmigración, el conocimiento del catalán es más alto en aquellas comarcas que reciben una proporción mayor de inmigrantes castellanoparlantes, lo cual indicaría que podría existir una «complementariedad» entre el castellano y el catalán debido a su proximidad lingüística. Al mismo tiempo, la proporción de extranjeros entre los inmigrantes tiene un impacto negativo sobre el conocimiento del catalán, debido probablemente a la más reciente llegada de los inmigrantes extranjeros y a una mayor distancia lingüística media entre sus lenguas maternas y el catalán. Por último, el conocimiento del catalán de los inmigrantes es mayor en las comarcas pirenaicas y menor en el área metropolitana de Barcelona.

En segundo lugar, analizamos el conocimiento del catalán de los inmigrantes nacidos en el resto de España, con resultados muy parecidos en 2001 y 1986. Como para el conjunto de los inmigrantes, seguimos obteniendo que la proporción de nacidos en Cataluña y la proporción de inmigrantes tienen respectivamente un impacto positivo y negativo sobre la variable endógena. Sin embargo, contrariamente a lo que ocurría para los inmigrantes en su conjunto, para los inmigrantes del resto de España la segunda variable es más significativa que la primera, en particular en 1986. En otras palabras, los inmigrantes del resto de España tienen un buen conocimiento del 
catalán sobre todo en aquellas comarcas en las que la «fuerza de monopolio» del castellano es relativamente baja, por ser baja la proporción de inmigrantes. La asimetría observada para estos dos grupos de inmigrantes podría explicarse de la forma siguiente: la fuerza de monopolio de la lengua materna de los inmigrantes del resto de España es elevada porque la inmensa mayoría tienen la misma lengua materna, mientras que el fraccionamiento de las lenguas maternas de los inmigrantes en su conjunto reduce la significatividad de la variable «proporción de inmigrantes». El resto de los resultados es muy parecido al del conjunto de los inmigrantes: la variable «nacionalismo catalán» es menos significativa que los factores económicos, y el conocimiento del catalán de los inmigrantes del resto de España es mayor en las zonas agrícolas y menor en el área metropolitana de Barcelona.

Por último, estudiamos el conocimiento del catalán en 2001 de los inmigrantes nacidos fuera de España. Dado que su llegada se concentra a partir de los años noventa, mucho después que las llegadas de inmigrantes del resto de España, consideramos como variable dependiente el conocimiento del catalán de los residentes nacidos en España en 1986. Esta variable tiene en algunas especificaciones un impacto positivo sobre la variable endógena, pero, contrariamente a lo que ocurría con los inmigrantes del resto de España, su impacto es menos significativo que el de la variable «nacionalismo catalán», lo cual podría indicar un predominio de los factores ideológicos para este grupo de inmigrantes. Otras dos variables que tienen un impacto muy significativo (respectivamente positivo y negativo) son el nivel de estudios de los inmigrantes y la densidad de población de la comarca. La interpretación del impacto positivo del nivel de estudios parece sencilla: aquellos inmigrantes con un nivel de estudios mayor tendrían ceteris paribus menos dificultades para aprender una lengua adicional debido por ejemplo a su mayor familiaridad con las técnicas de aprendizaje. Para interpretar el impacto negativo de la densidad de población, nos basamos en la Estadística d'Usos Lingüístics a Catalunya, que indica que las regiones con mayor densidad están caracterizadas por un mayor uso relativo del castellano frente al catalán, que podría por lo tanto disminuir los incentivos de aprendizaje del catalán para los inmigrantes. Finalmente, obtenemos también que la proporción de inmigrantes procedentes del continente americano tiene en algunos casos un impacto positivo sobre la variable endógena, en lo que entendemos como un nuevo ejemplo de complementariedad entre el castellano y el catalán.

Nuestro modelo está relacionado con una literatura reciente sobre aprendizaje de lenguas, y en particular con Rendon (2006), Ortega y Verdugo (2006), Church y King (1993) y Lazear (1999). A partir de datos censales de 1991 y 1996 para Cataluña, Rendon (2006) muestra que la probabilidad de estar empleado de un individuo aumenta entre 3 y 5 puntos porcentuales si sabe hablar y leer catalán. Ortega y Verdugo (2006) muestran para el caso de los inmigrantes en Canadá que la «fuerza de atracción» del inglés es mayor que la del francés. Church y King (1993) utilizan la teoría de juegos para estudiar por qué en un país con dos lenguas es más probable que los miembros de la minoría sean bilingües. Lazear (1999), con datos para Estados Uni- 
dos, muestra que es más probable que los inmigrantes pertenecientes a grupos lingüísticos pequeños aprendan inglés.

El resto del artículo se organiza de la forma siguiente. En la sección 2, presentamos los datos utilizados. La sección 3 se dedica a estudiar los determinantes del nivel de catalán de los inmigrantes en su conjunto, mientras que las secciones 4 y 5 presentan este análisis para los inmigrantes nacidos en el resto de España y fuera de España, respectivamente. Finalmente, en la sección 6 presentamos algunas conclusiones sobre este análisis.

\section{Los datos}

Este artículo presenta un estudio empírico de los determinantes del conocimiento del catalán por parte de los residentes en Cataluña nacidos fuera de esta comunidad autónoma, a los que nos referiremos como «inmigrantes». Según el Censo General de Población y Vivienda de 1986, 5.978.638 personas residían en Cataluña, de las que 65,25\% había nacido en Cataluña, 33,04\% en el resto de España, y 1,71\% fuera de España. En 2001, la población era de 6.343 .110 habitantes, con 67,88\% nacidos en Cataluña, 26\% nacidos en el resto de España y 6,12\% nacidos fuera de España.

Nuestra variable endógena es la proporción de inmigrantes en cada comarca que declara saber hablar catalán. Escogemos por lo tanto un criterio de capacidad de utilización oral del catalán en vez de un criterio de comprensión oral o de capacidad de escritura o lectura. La razón es que este criterio podría considerarse como intermedio: en efecto, por una parte, la proximidad lingüística del castellano y el catalán hace que la comprensión del catalán sea fácilmente alcanzable. En el extremo opuesto, un criterio de lectura o escritura excluiría a los individuos que no saben leer ni escribir, y daría además probablemente una idea sesgada del conocimiento del catalán, ya que una proporción importante de los nacidos en Cataluña conocen oralmente el catalán pero no han aprendido a escribirlo.

Los datos provienen de los Censos Generales de Población y Vivienda de 1986 y 2001, que incluyen para los residentes en la Comunidad Autónoma de Cataluña en el Cuestionario de hogar un apartado adicional sobre el conocimiento del catalán, con las siguientes casillas: (i) «No lo entiende/No l'entén», (ii) «Lo entiende/L'entén», (iii) «Lo entiende y lo sabe leer/L'entén i el sap llegir», (iv) «Lo sabe hablar/El sap parlar», (v) «Lo sabe hablar y leer/El sap parlar i llegir», (vi) «Lo sabe hablar, leer y escribir/El sap parlar, llegir i escriure». En el sitio internet del Institut d'Estadística de Catalunya ${ }^{1}$ se ofrecen datos sobre el número de individuos en cada comarca que «entiende el catalán» (correspondiente al número de individuos que han respondido (ii), (iii), (iv), (v) o (vi) en la encuesta), «lo sabe hablar» (suma de las categorías (iv), (v) y (vi)), «lo sabe leer» (suma de las categorías (iii), (v) y (vi)), «lo sabe escribir» (ca-

1 http://www.idescat.net/ 
tegoría (vi)) y «no lo entiende» (categoría (i)). El número de individuos que sabe hablar catalán en cada comarca aparece también desglosado según el lugar de nacimiento de los individuos. Más exactamente, se proporcionan datos para los nacidos en el extranjero, y para los nacidos en España según la provincia de nacimiento (sólo para los nacidos en Cataluña) o según la comunidad autónoma de nacimiento. La tabla 1 resume para 1986 y 2001 los niveles medios de conocimiento oral del catalán, así como las comarcas en las que estos niveles son máximos y mínimos, para los diferentes grupos de individuos considerados, es decir, el conjunto de los residentes, los individuos nacidos en Cataluña, los inmigrantes en su conjunto, y los inmigrantes según su nacimiento dentro o fuera de España. Como se puede observar, el conocimiento oral del catalán ha aumentado del $64,2 \%$ al 74,5\% entre 1986 y 2001 , y este aumento se produce para todos los grupos de individuos excepto para los inmigrantes nacidos fuera de España. Esta última característica es probablemente debida a una composición muy diferente en 1986 y 2001 de los inmigrantes nacidos fuera de España. En 1986, el nivel de estudios de los inmigrantes nacidos fuera de España era superior al de los individuos nacidos en Cataluña (por ejemplo, 21,9\% de los inmigrantes no sabía leer y escribir, o no tenía estudios, por 36,3\% de los nacidos en Cataluña), mientras que en 2001 se da la relación opuesta (15,4\% de los inmigrantes no había acabado los estudios primarios, por 7,62\% de los nacidos en Cataluña) ${ }^{2}$.

El resto de los datos de la Tabla 1 parecen poder interpretarse fácilmente. Por una parte, en 1986 y 2001, los nacidos en Cataluña tienen un mayor conocimiento del catalán que los inmigrantes debido a que muy pocos inmigrantes tienen el catalán como lengua materna y a que el catalán es la lengua vehicular de la enseñanza en las escuelas. Por otra, en 2001, los inmigrantes del resto de España tienen un nivel de catalán mayor que los inmigrantes nacidos fuera de España probablemente debido a su estancia más larga en Cataluña y a la mayor proximidad de su lengua materna (en una proporción muy alta, el castellano) al catalán. Finalmente, puede observarse también que los menores niveles de conocimiento del catalán se dan en el área metropolitana de Barcelona (en particular, en el Baix Llobregat) y que los niveles más altos se dan en comarcas con un fuerte peso de la agricultura. Se puede observar también que en 2001, la comarca con el menor conocimiento del catalán entre los nacidos en Cataluña (Baix Llobregat) es también la comarca con el menor conocimiento del catalán entre los inmigrantes. Presentamos a continuación unos gráficos para estudiar este tipo de correlación.

El gráfico 1 presenta para las 41 comarcas la proporción de inmigrantes que saben hablar catalán en función de la proporción de nacidos en Cataluña que saben hablar catalán. Puede observarse fácilmente que la correlación entre las dos variables es positiva. Las proporciones más bajas de conocimiento del catalán entre los inmigrantes (con valores entre 37,5\% y 42\%) se dan en tres comarcas del área metropo-

2 Otra hipótesis que no podemos contrastar es que una fracción significativa de los inmigrantes nacidos fuera de España sean hijos de exiliados republicanos que tenían como lengua materna el catalán y se habrían instalado en Cataluña. 


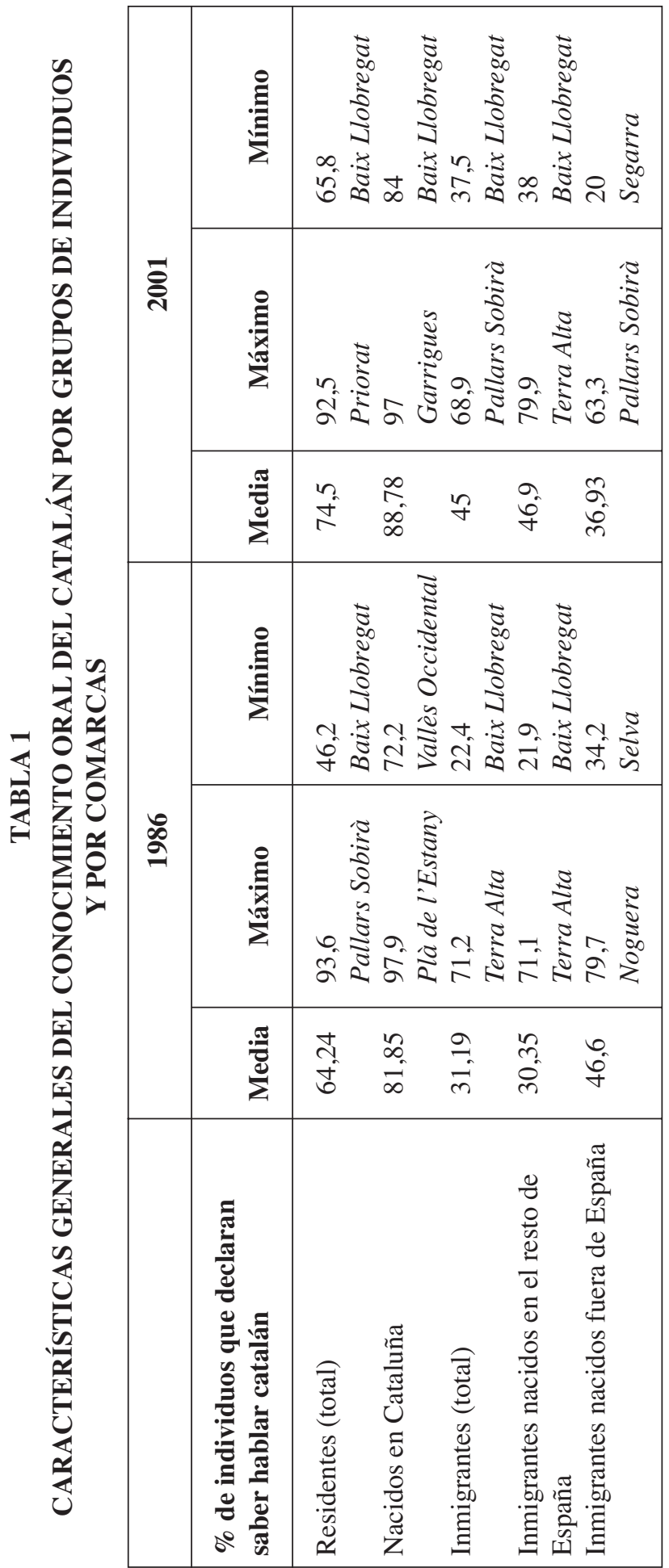


litana de Barcelona (Baix Llobregat, Vallès Occidental y Barcelonès). Estas tres comarcas tienen también los porcentajes más bajos de catalanoparlantes entre los nacidos en Cataluña (con valores entre $83 \%$ y $87 \%$ ). En el extremo opuesto, las cinco comarcas con un mayor conocimiento del catalán entre los inmigrantes (cuatro de la provincia de Tarragona, y una del Pirineo leridano) están caracterizadas por un conocimiento del catalán superior al $94 \%$ entre los nacidos en Cataluña.

El mismo tipo de correlación positiva puede observarse si se compara la proporción de catalanoparlantes entre les nacidos en Cataluña y los inmigrantes nacidos en el resto de España (ver gráfico 2).

En cambio, el gráfico 3 indica que la proporción comarcal de catalanoparlantes dentro de los individuos nacidos en Cataluña no parece estar correlacionada positivamente con la proporción de inmigrantes nacidos fuera de España que declaran hablar catalán.

\section{Conocimiento del catalán del conjunto de los inmigrantes}

Estudiamos en primer lugar los determinantes del conocimiento del catalán para el conjunto de los inmigrantes. La variable dependiente corresponde a la transformación logística de la proporción de inmigrantes que declaran saber hablar catalán ${ }^{3}$. Las estimaciones se hacen por mínimos cuadrados ordinarios (MCO). La Tabla 2 presenta los resultados de las regresiones cuando se atribuye el mismo peso a cada una de las comarcas, mientras que en las regresiones presentadas en la Tabla 3 el peso atribuido a cada comarca depende de su población. Atribuir el mismo peso a todas las comarcas puede ser problemático, porque tienen un número total de inmigrantes muy diferentes: tomando los casos extremos, Pallars Sobirà (en el Pirineo leridano) contaba con 831 personas nacidas fuera de Cataluña, mientras que en el Barcelonès había 756.990 inmigrantes. Al mismo tiempo, atribuir pesos a las comarcas según su población lleva a asignar un peso muy bajo a muchas comarcas, dadas las asimetrías existentes: tomando una vez más los casos extremos, Alta Ribagorça (también en el Pirineo leridano) tenía 3.477 habitantes, por un poco más de dos millones el Barcelonès. Elegimos por lo tanto presentar los resultados para los dos tipos de regresiones, y veremos además que en general los resultados no cambian según el método utilizado.

La regresión (1) de las tablas 2 y 3 muestra, como indicaba el gráfico 1, que existe una relación positiva entre el nivel de catalán de los nacidos en Cataluña y el nivel de catalán de los inmigrantes. Con los dos métodos, esta variable es significativa al $1 \%$. Este resultado podría interpretarse de forma muy simple a través un modelo teórico: dado que el aprendizaje de una lengua es costoso, suponiendo que los individuos necesitan hablar la misma lengua para las actividades productivas o de intercambio, es más probable que los inmigrantes elijan aprender el catalán si hay más individuos

3 Es decir, $\ln (y /(1-y))$, donde $y$ es la proporción de inmigrantes que declaran saber hablar catalán. 


\section{GRÁFICO 1}

CONOCIMIENTO DEL CATALÁN POR COMARCAS:

NACIDOS EN CATALUÑA E INMIGRANTES (2001)

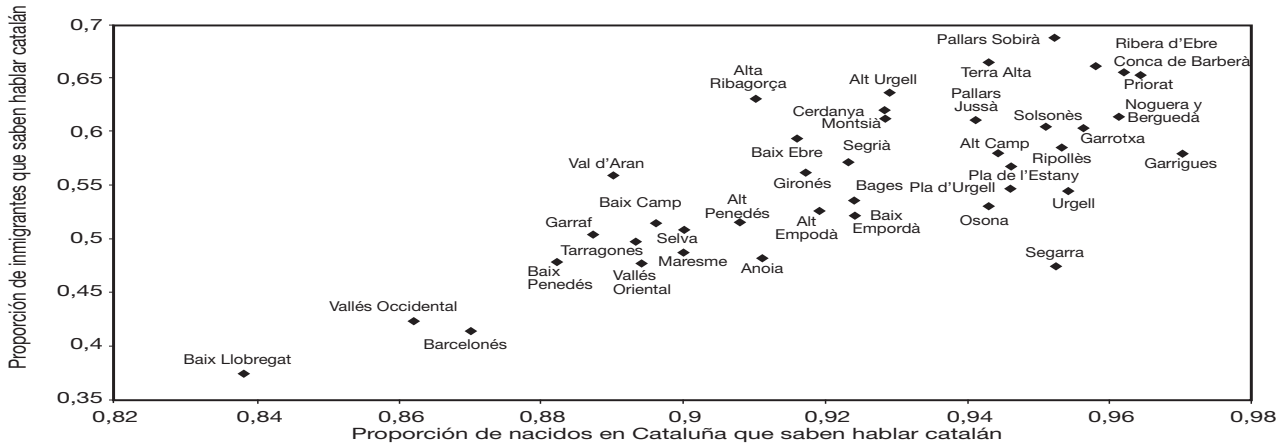

\section{GRÁFICO 2}

CONOCIMIENTO DEL CATALÁN POR COMARCAS:

NACIDOS EN CATALUÑA E INMIGRANTES DEL RESTO DE ESPAÑA (2001)

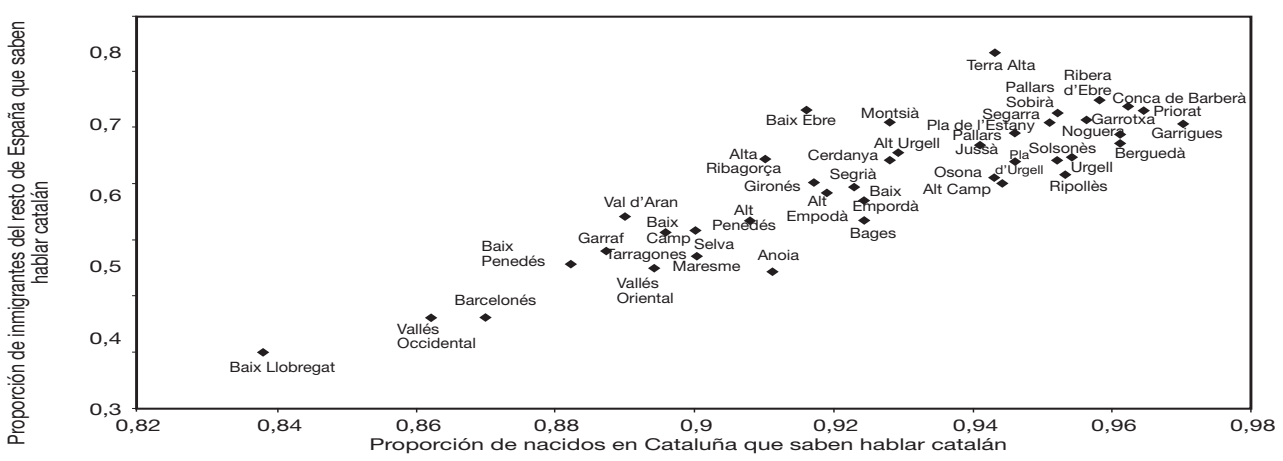

\section{GRÁFICO 3}

CONOCIMIENTO DEL CATALÁN POR COMARCAS:

NACIDOS EN CATALUÑA E INMIGRANTES DE FUERA DE ESPAÑA (2001)

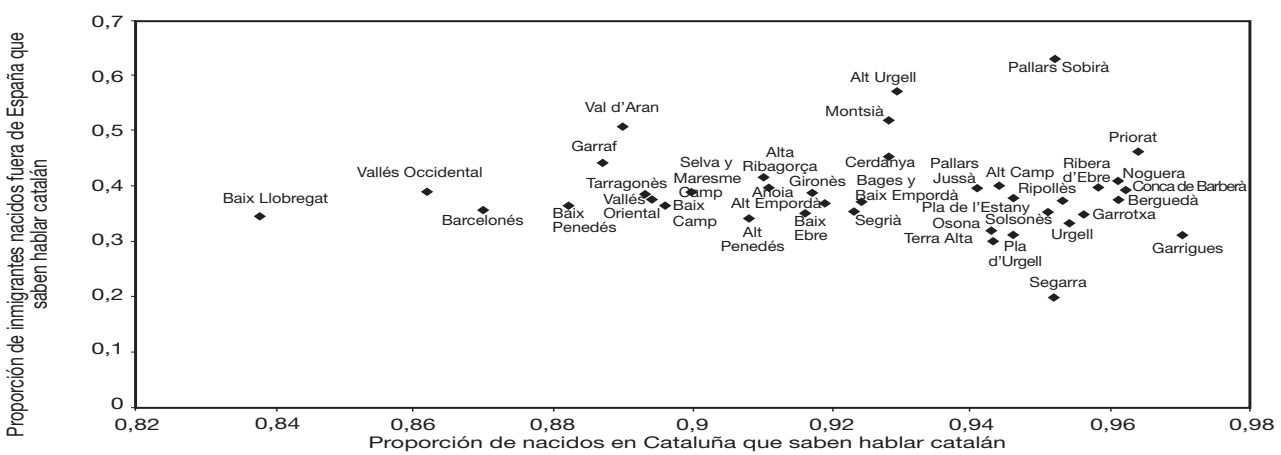




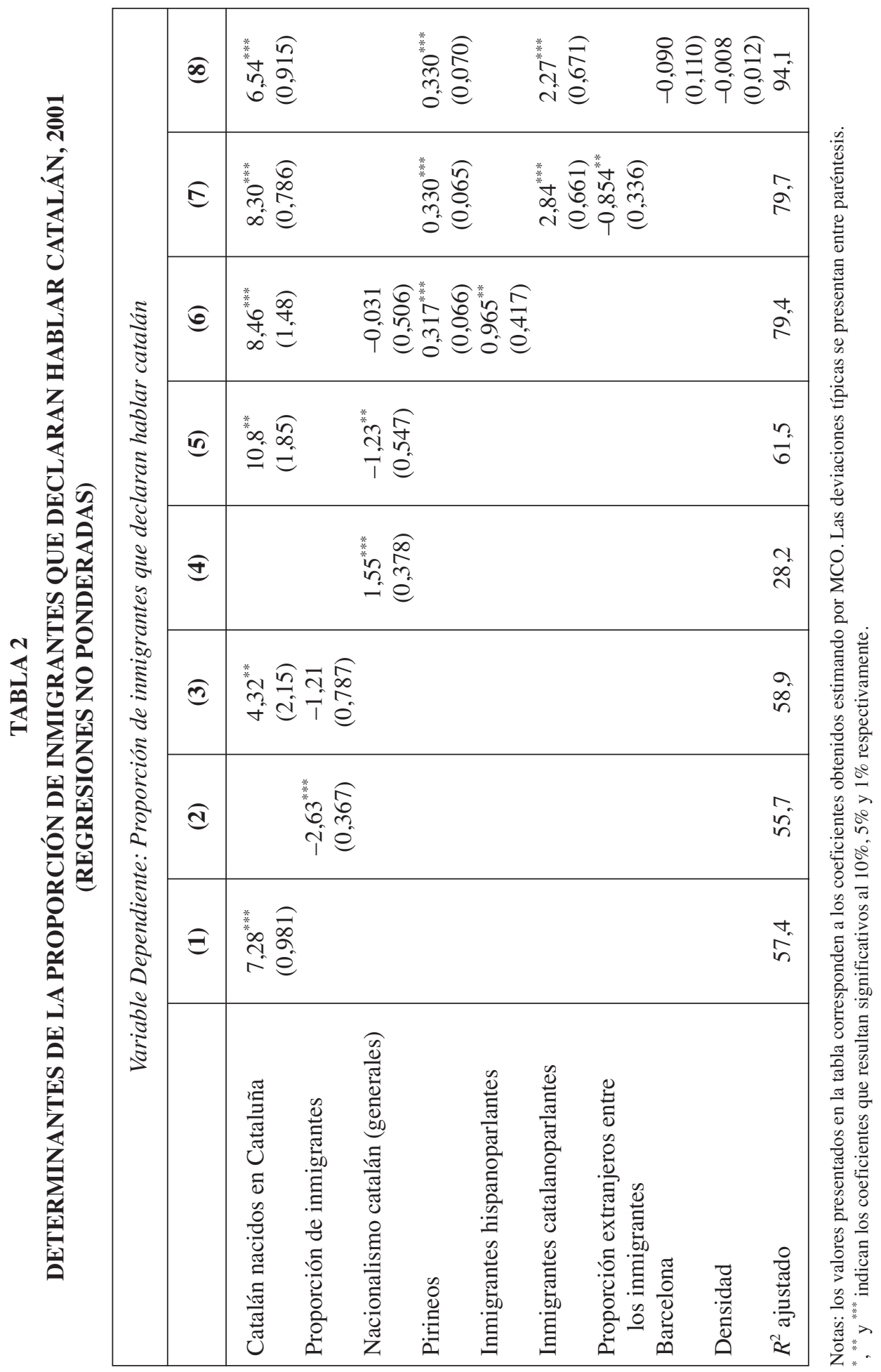


nacidos en Cataluña que hablan esta lengua (ver por ejemplo Ortega y Verdugo (2006) para un modelo de este tipo).

La regresión (2) estudia el impacto de la proporción de inmigrantes en la comarca sobre la proporción de inmigrantes que declaran hablar catalán. Encontramos que existe una relación negativa entre estas dos variables con, de nuevo, una significatividad al $1 \%$. Este resultado podría interpretarse de la forma siguiente: dado que la mayor parte de los inmigrantes hablan castellano (puesto que como hemos visto un poco más del 80\% vienen del resto de España, y un 37\% de los inmigrantes extranjeros poseen una nacionalidad de un país americano), invertir en el aprendizaje del catalán tiene asociada una ganancia neta más baja ceteris paribus en las comarcas en las que viven muchos inmigrantes. En otras palabras, un inmigrante que se instala en una comarca en la que hay muchos inmigrantes podrá producir o intercambiar con muchas personas sin necesidad de aprender catalán.

En la tercera regresión, utilizamos como variables explicativas tanto la proporción de nacidos en Cataluña que declaran hablar catalán como la proporción de inmigrantes. Vemos que la proporción de nacidos en Cataluña que declaran hablar catalán sigue teniendo un impacto positivo y significativo sobre el nivel de catalán de los inmigrantes, mientras que la proporción de inmigrantes pasa a ser no significativa. Este resultado podría indicar que un mayor aprendizaje del catalán por parte de los inmigrantes está más relacionado con una atractividad alta del catalán (i.e. la posibilidad de producir o intercambiar mucho si habla catalán), que con una atractividad baja del castellano (i.e. el hecho de que produzca poco si habla castellano y no paga el coste de aprender catalán).

El aprendizaje de una lengua ${ }^{4}$ podría ser a priori el resultado de factores ideológicos, y no sólo o principalmente de factores económicos. En Cataluña, los partidos nacionalistas catalanes han puesto el énfasis en la defensa y desarrollo del uso del catalán como elemento de una «identidad nacional» catalana. Para intentar estudiar el impacto del nacionalismo, definimos una variable «nacionalismo catalán», correspondiente a la suma comarcal de los porcentajes de votos obtenidos en las elecciones por Convergència i Unió y Esquerra Republicana de Catalunya. En principio, dado que los partidos nacionalistas catalanes obtienen sistemáticamente un porcentaje de votos mayor en las elecciones autonómicas, utilizamos dos versiones de esta variable, la primera basada en los resultados de las elecciones autonómicas de 1999, y la segunda en los resultados de las elecciones generales del 2000. Sin embargo, la correlación inter-comarcal de estas dos variables es muy elevada $(0,982)$, por lo cual los resultados con las dos variables son muy parecidos. La regresión (4) muestra que la variable «nacionalismo catalán» está correlacionada positivamente con la proporción de inmigrantes que declaran hablar catalán. El coeficiente correspondiente es significativo al 1\%, aunque al mismo tiempo el ajustado es menor que en las regresiones precedentes. El signo del coeficiente no es sorprendente si se tiene en cuenta que existe una fuerte correlación positiva entre la declaración del conocimiento del

4 O la tendencia a declarar que tal lengua se conoce. 


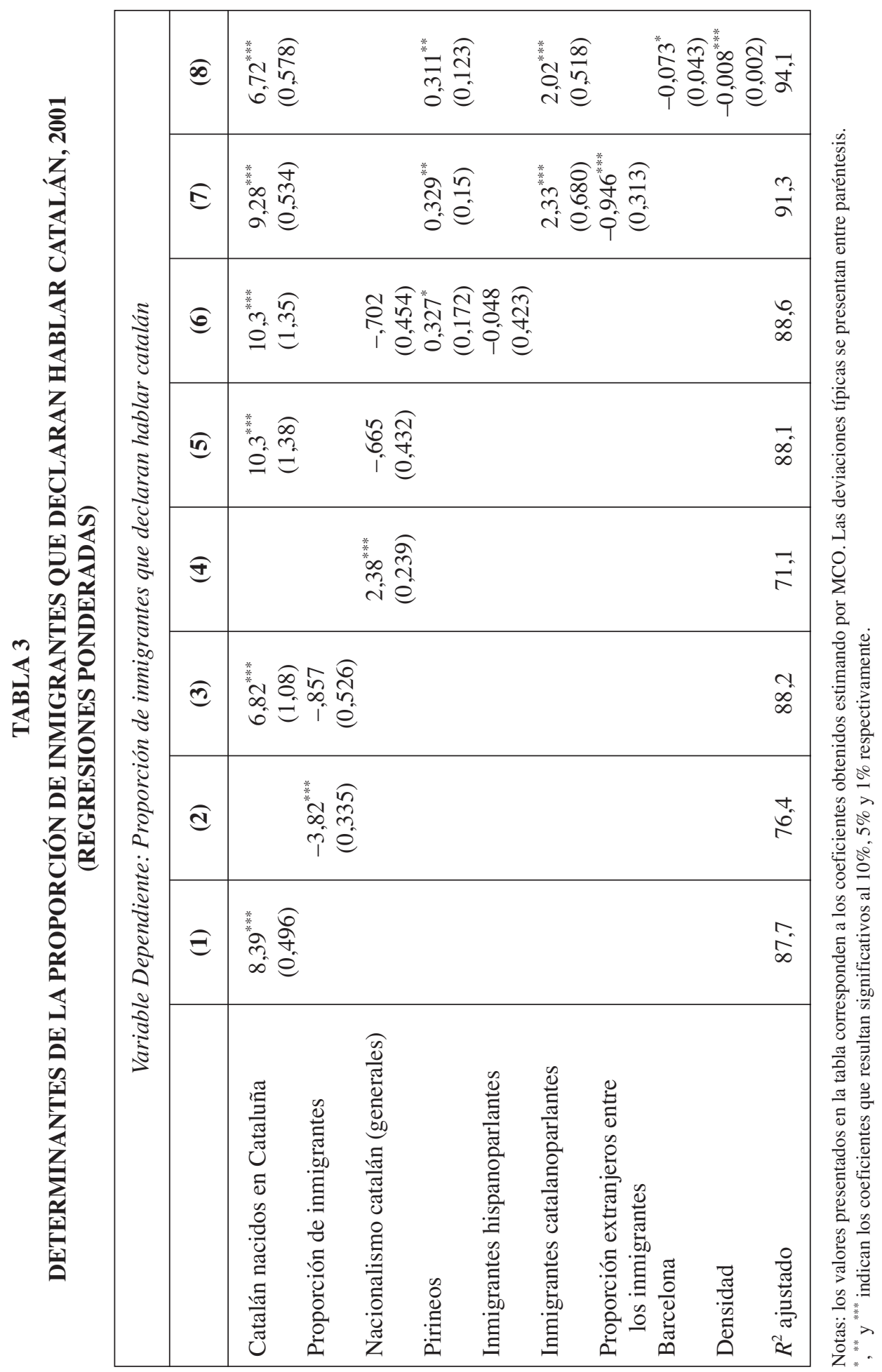


catalán por parte de las personas nacidas en Cataluña y el voto a partidos nacionalistas catalanes por parte los residentes con derecho a voto ( 0.88 y 0.86 para los resultados de las elecciones autonómicas y generales respectivamente).

Sin embargo, el signo asociado a la variable «nacionalismo catalán» cambia cuando se añade como regresor la proporción de nacidos en Cataluña que declaran hablar catalán (regresión (5) de las tablas 2 y 3). En efecto, mientras que el nivel de catalán de los nacidos en Cataluña sigue teniendo un impacto positivo y significativo en el nivel de catalán de los inmigrantes, la variable «nacionalismo catalán» pasa a tener un impacto negativo sobre el nivel de catalán de los inmigrantes, aunque el coeficiente es significativo sólo en el caso de las regresiones no ponderadas por la población (tabla 1). Interpretar este resultado como un impacto negativo de la presión ideológica nacionalista sobre el aprendizaje del catalán por parte de los inmigrantes parece un poco aventurado, ya que la relación entre las dos variables podría también interpretarse en la otra dirección: la falta de aprendizaje del catalán por parte de los inmigrantes podría reforzar el voto nacionalista.

En la regresión (6) añadimos dos variables, una variable indicativa para las comarcas pirenaicas ${ }^{5}$ y una variable que indica la proporción de inmigrantes castellanoparlantes entre los inmigrantes. La primera variable se introduce porque las comarcas pirenaicas están caracterizadas por una proporción de inmigrantes baja respecto a la media ${ }^{6}$, un hábitat disperso con obstáculos geográficos y un peso de la agricultura superior a la media. Tanto para la tabla 1 como para la tabla 2, esta variable tiene asociado un coeficiente positivo y significativo. Una interpretación de este resultado podría ser la siguiente: al reducir los obstáculos geográficos el conjunto de individuos con el que se puede producir y ser éstos en una proporción importante catalanoparlantes, los incentivos para el aprendizaje del catalán son mayores. Por su parte, la proporción de inmigrantes castellanoparlantes tiene un impacto positivo sobre el conocimiento del catalán por parte de los inmigrantes, pero el coeficiente es significativo sólo en el caso de la regresión no ponderada. Esta variable se construye calculando la proporción de inmigrantes con nacionalidad española o de un país americano con respecto al número total de inmigrantes ${ }^{7}$. El coeficiente es positivo y significativo, pero sólo en la regresión no ponderada. Este resultado podría indicar que la pequeña distancia lingüística existente entre el castellano y el catalán hace más probable el aprendizaje del catalán por parte de los inmigrantes castellanoparlantes que por parte de inmigrantes con otras lenguas maternas más lejanas del catalán.

En la regresión (7), se confirma el impacto positivo y significativo de la proporción de nacidos en Cataluña que habla catalán y de la variable indicativa «Pirineos».

La definición de este grupo de comarcas es del IDESCAT («Comarques del Alt Pirineu i Aran»). Estas comarcas son Alt Urgell, Alta Ribagorça, Cerdanya, Pallars Jussà, Pallars Sobirà y Val d'Aran.

6 La proporción media (ponderada por la población) es del $21.6 \%$ para las comarcas pirenaicas, frente al 32\% para el conjunto de Cataluña. Una excepción es el Val d'Aran, con una proporción de $33 \%$ de residentes nacidos fuera de Cataluña.

7 En 2001, 66.837 inmigrantes de nacionalidad americana residían en Cataluña. El número de norteamericanos (2.153) era reducido, y los brasileños no constituían un grupo importante. 
Añadimos además en esta regresión la proporción de inmigrantes catalanoparlantes en el conjunto de los inmigrantes y la proporción de extranjeros entre los inmigrantes. A falta de datos concretos sobre la lengua materna de los inmigrantes, suponemos que todos los inmigrantes nacidos en la Comunidad Valenciana y en las Baleares hablan catalán, lo cual sobre-estima sin duda el número de inmigrantes catalanoparlantes. Según lo esperado, la proporción comarcal de inmigrantes provinientes de estas dos comunidades autónomas tiene un impacto positivo (y significativo al 1\%) sobre la proporción comarcal de inmigrantes que declaran hablar catalán. Al mismo tiempo, la proporción de extranjeros entre los inmigrantes tiene un impacto negativo y significativo sobre la variable endógena. Interpretamos este resultado de nuevo en clave de distancia lingüística, puesto que, contrariamente a la inmensa mayoría de los inmigrantes de otras regiones españolas, una proporción importante de los inmigrantes extranjeros no tienen el castellano como lengua materna.

Finalmente, en la regresión (8) introducimos una variable indicativa para Barcelona ${ }^{8}$ y la densidad de población. Tal y como esperábamos, el signo es negativo, pero no es siempre significativo. La densidad de población tiene un impacto negativo sobre la proporción de inmigrantes que declaran hablar catalán, pero sólo en el caso de la regresión ponderada por la población. Este resultado podría ser debido a que la densidad corresponde a la población dividida por la superficie, y que se está precisamente ponderando por la población. Sin embargo, veremos en la sección 4 que este tipo de resultado se obtiene también para el caso de los inmigrantes extranjeros en las regresiones no ponderadas. Una interpretación de este resultado podría estar basada en la uso relativo del catalán y el castellano en las comarcas según su densidad. Los datos comarcales incluyen sólo información sobre el conocimiento del catalán, por lo cual no podemos saber si el conocimiento o uso del castellano es mayor o menor que el del catalán en cada una de las comarcas. Para el caso de Cataluña, existen datos sobre la «lengua habitual» de los individuos, pero a un nivel de agregación superior al comarcal, para los llamados «àmbits territorials» ${ }^{9}$. A partir de estos datos, si se clasifican en orden decreciente los ámbitos territoriales según el porcentaje de individuos que utilizan el catalán como lengua habitual, se obtendrá una clasificación casi idéntica a la que se obtiene si se clasifican en orden creciente los ámbitos territoriales según su densidad ${ }^{10}$. En otras palabras, de forma casi sistemá-

\footnotetext{
8 Esta variable toma valor igual a 1 para las comarcas pertenecientes al área metropolitana de Barcelona, es decir Baix LLobregat, Barcelonès, Maresme y Vallès Occidental.

9 IDESCAT (2003). Las comarcas se reagrupan en siete ámbitos territoriales: Ambit metropolità, Comarques gironines, Camp de Tarragona, Terres de l'Ebre, Ambit de Ponent, Comarques Centrals y Alt Pirineu i Aran.

10 La clasificación decreciente según el porcentaje de individuos que utilizan el catalán como lengua habitual es la siguiente: 1) Terres de l'Ebre 89,4\%,2) Ambit de Ponent 76,5\%,3) Comarques Centrals, $72,2 \%, 4)$ Alt Pirineu i Aran 71,7\%,5) Comarques gironines 70,3\%,6) Camp de Tarragona 54,4\%, 7) Ambit metropolità 41,5. La clasificación creciente de la densidad es idéntica salvo en lo que concierne al Alt Pirineu i Aran: 1) Alt Pirineu i Aran, 2) Terres de l'Ebre, 3) Ambit de Ponent, 4) Comarques Centrals, 5) Comarques gironines, 6) Camp de Tarragona, 7) Ambit metropolità. Existe también una relación inversa entre la densidad de población y la proporción de individuos que se dirigirían a una persona desconocida en catalán.
} 
tica, las regiones con menor densidad utilizan más el catalán, y esto podría explicar el impacto negativo de la variable «densidad» en el conocimiento del catalán por parte de los inmigrantes. Sin embargo, los datos sobre «lengua habitual» incluyen a todos los residentes en Cataluña sin información sobre su lugar de nacimiento, y por lo tanto no se pueden utilizar en las regresiones.

En resumen, las conclusiones principales de esta sección son las siguientes: (i) el conocimiento del catalán por parte de los nacidos en Cataluña favorece el conocimiento del catalán por parte de los inmigrantes (ii) las zonas pirenaicas están caracterizadas por un mayor conocimiento del catalán por parte de los inmigrantes (iii) en las comarcas con un mayor porcentaje de inmigrantes de fuera de España, el nivel de catalán de los inmigrantes es menor (iv) en las comarcas con una mayor proporción de inmigrantes provinientes de la Comunidad Valenciana o de Baleares, el nivel de catalán de los inmigrantes es mayor.

\section{Conocimiento del catalán de los inmigrantes del resto de España}

Realizamos a continuación el mismo tipo de regresiones pero para estudiar esta vez específicamente el nivel de conocimiento del catalán de los inmigrantes del resto de España. Los resultados serán presentados resaltando las diferencias y similitudes con los resultados obtenidos para el conjunto de los inmigrantes. Como es bien conocido, los movimientos migratorios más importantes procedentes del resto de España se produjeron en los años cincuenta y sesenta, mientras que la inmigración proviniente de fuera de España empezó a ser importante a partir de los años noventa.

Una opción interesante habría sido estudiar el nivel de catalán de los inmigrantes del resto de España un cierto número de años después de su llegada a Cataluña en función del nivel de catalán de los nacidos en Cataluña en el momento de la migración. Sin embargo, los primeros datos censales sobre la capacidad de expresión en catalán son de 1986 (ver Capellades, 2001), y no disponemos por lo tanto del nivel de catalán de los nacidos en Cataluña en los años de fuerte inmigración del resto de España.

Para explicar el nivel de catalán de los inmigrantes del resto de España en 2001, utilizamos como variable explicativa el nivel de catalán en ese mismo año de los nacidos en Cataluña. Esto podría ser problemático si el nivel de catalán de los nacidos en Cataluña depende del nivel de catalán de los inmigrantes. Comparando los censos de 1986 y 2001, sabemos en efecto que el nivel de catalán de tanto los nacidos en Cataluña como de los inmigrantes del resto de España ha aumentado entre 1986 y 2001 (ver Tabla 1), pero este artículo no analiza las causas de esta evolución. Sin embargo, sí mostraremos que los determinantes del conocimiento oral del catalán por parte de los inmigrantes del resto de España son bastante parecidos en 2001 y en 1986.

Dado que las oleadas de inmigración extranjera son posteriores a las que provenían del resto de España, no incluiremos entre las variables explicativas del nivel de catalán de los inmigrantes del resto de España variables relacionadas con la inmigración extranjera. En cambio, al estudiar el nivel de catalán de los inmigrantes extran- 
jeros en la próxima sección, sí utilizaremos variables relacionadas con la inmigración del resto de España.

Como en las regresiones para el conjunto de los inmigrantes, el nivel de conocimiento del catalán de los nacidos en Cataluña tiene un impacto positivo sobre el nivel de catalán de los inmigrantes del resto de España. La única diferencia reside en la más baja significatividad de esta variable en las regresiones no ponderadas. En particular, esta variable es significativa sólo al 10\% en las regresiones (5) y (7) de la tabla 4, y se convierte en no significativa en la la regresión (8) de esa misma tabla. Este resultado tiene una interpretación sencilla cuando se comprueba que en conjunto de las regresiones de las tablas 4 y 5 , la variable «proporción de inmigrantes españoles» tiene siempre un impacto negativo sobre el nivel de conocimiento de catalán de los inmigrantes, y es en la mayor parte de los casos significativa al 1\% (en particular, en las regresiones (5), (7) (8) de la tabla 4 a las que nos referíamos antes). En efecto, estos resultados parecen indicar que, contrariamente a lo que ocurre para los inmigrantes en su conjunto, un mayor conocimiento del catalán por parte de los inmigrantes del resto de España está más relacionado con una atractividad baja del castellano (la presencia de una pequeña proporción de inmigrantes en la comarca) que con una atractividad alta del catalán (un nivel alto de catalán de los nacidos en Cataluña).

Dos variables que no eran significativas para el conjunto de los inmigrantes pasan a serlo cuando se consideran sólo los inmigrantes del resto de España. Por una parte, la variable «agricultura», medida como la proporción de la población ocupada en el sector agrícola, tiene un impacto positivo y siempre significativo sobre la proporción de inmigrantes del resto de España que declaran hablar catalán. Una interpretación posible de este resultado sería que en las comarcas más rurales, el bilingüismo o el conocimiento del castellano podrían ser más bajos, y por lo tanto los inmigrantes tendrían más incentivos para aprender el catalán. Sin embargo, esta interpretación no puede apoyarse empíricamente porque no existen datos de conocimiento del castellano a nivel comarcal. La otra variable que pasa a ser significativa es la variable indicativa «Barcelona», manteniendo siempre un impacto negativo sobre el nivel de catalán de los inmigrantes.

Finalmente, el papel jugado por las variables «nacionalismo catalán» ${ }^{11} \mathrm{y}$ densidad es el mismo que en la sección precedente, y la variable inmigrantes catalanoparlantes sigue teniendo un impacto positivo, pero no es siempre significativa.

Las tablas A1 y A2 del anexo presentan las mismas regresiones realizadas con los datos del censo de 1986. El nivel de los catalán de los nacidos en Cataluña tiene también en 1986 un impacto positivo sobre el catalán de los inmigrantes del resto de España, pero la significatividad de la variable es menor ${ }^{12}$ y el valor del coeficiente es menor al de 2001 cuando la variable es significativa.

11 En este caso, el resultado de las elecciones generales, pero se obtienen unos resultados parecidos si se utilizan como en la sección precedente los resultados de las elecciones autonómicas.

${ }_{12}$ No es significativa por ejemplo en las especificaciones (5), (6), (7) y (8) de la regresión, contrariamente a los resultados obtenidos para 2001. Además, en la regresión (1), el $R^{2}$ ajustado es bastante menor en 1986 que en 2001, tanto en la regresión ponderada como en la no ponderada. 
La variable «nacionalismo catalán» sigue teniendo un impacto negativo como en 2001 en la ecuación (3), pero en 1986 el coeficiente es significativo. Sin embargo, como indicamos para los resultados de la Tabla 2 para el conjunto de los inmigrantes, no podemos descartar que un menor conocimiento del catalán por parte de los inmigrantes (en este caso, del resto de España) aumente el voto nacionalista catalán.

Por su parte, la proporción de inmigrantes del resto de España en la comarca sigue teniendo un impacto negativo y significativo sobre el conocimiento del catalán de los inmigrantes, con niveles de significatividad parecidos a los de 2001. Finalmente, se obtienen resultados parecidos para la densidad, mientras que la variable agricultura y Barcelona dejan de ser significativas en algunos casos, y en cambio el impacto positivo de la inmigración catalanoparlante pasa a ser más significativo.

En resumen, las conclusiones principales de esta sección son las siguientes: (i) para los inmigrantes del resto de España, la atractividad del castellano (medida por la proporción de inmigrantes del resto de España) podría ser un factor más importante que la atractividad del catalán (medidad por el nivel de catalán de los nacidos en Cataluña), aunque los dos factores parecen relevantes (ii) el conocimiento del catalán de los inmigrantes es mayor en las zonas rurales y menor en Barcelona. Los resultados para 1986 son parecidos a los de 2001.

\section{Conocimiento del catalán de los inmigrantes nacidos fuera de España}

En esta sección, estudiamos los determinantes del conocimiento del catalán por parte de los inmigrantes nacidos en el extranjero.

Las variables explicativas que utilizamos son en algunos casos diferentes de las utilizadas hasta ahora. En primer lugar, dado que la llegada de inmigrantes extranjeros a Cataluña se concentra a partir de los años noventa, mucho después que las llegadas de inmigrantes del resto de España, no consideramos como variable dependiente el conocimiento del catalán de los nacidos en Cataluña, sino el conocimiento del catalán de los residentes nacidos en España, es decir, del conjunto de los individuos que residían en Cataluña antes de la llegada de los inmigrantes extranjeros. Por la misma razón, utilizamos como variable dependiente la proporción de inmigrantes en su conjunto, y no sólo la proporción de inmigrantes españoles, como en el caso de la sección precedente.

En segundo lugar, medimos el conocimiento del catalán de los residentes nacidos en España (variable «catalán residentes españoles») en 1986, y no en 2001. La ventaja de utilizar los datos de 1986 es que de esta forma se garantiza la exogeneidad de la variable dependiente con respecto al nivel de catalán de los inmigrantes extranjeros en 2001. Por la misma razón, medimos la variable «nacionalismo catalán» en 1986. Más exactamente, corresponde a la media del porcentaje de voto obtenido por CiU y ERC en las elecciones autonómicas de 1984 y 1988.

En tercer lugar, el sitio del IDESCAT ofrece información desagregada del nivel de educación de los residentes en Cataluña según su nacimiento en España o en el ex- 


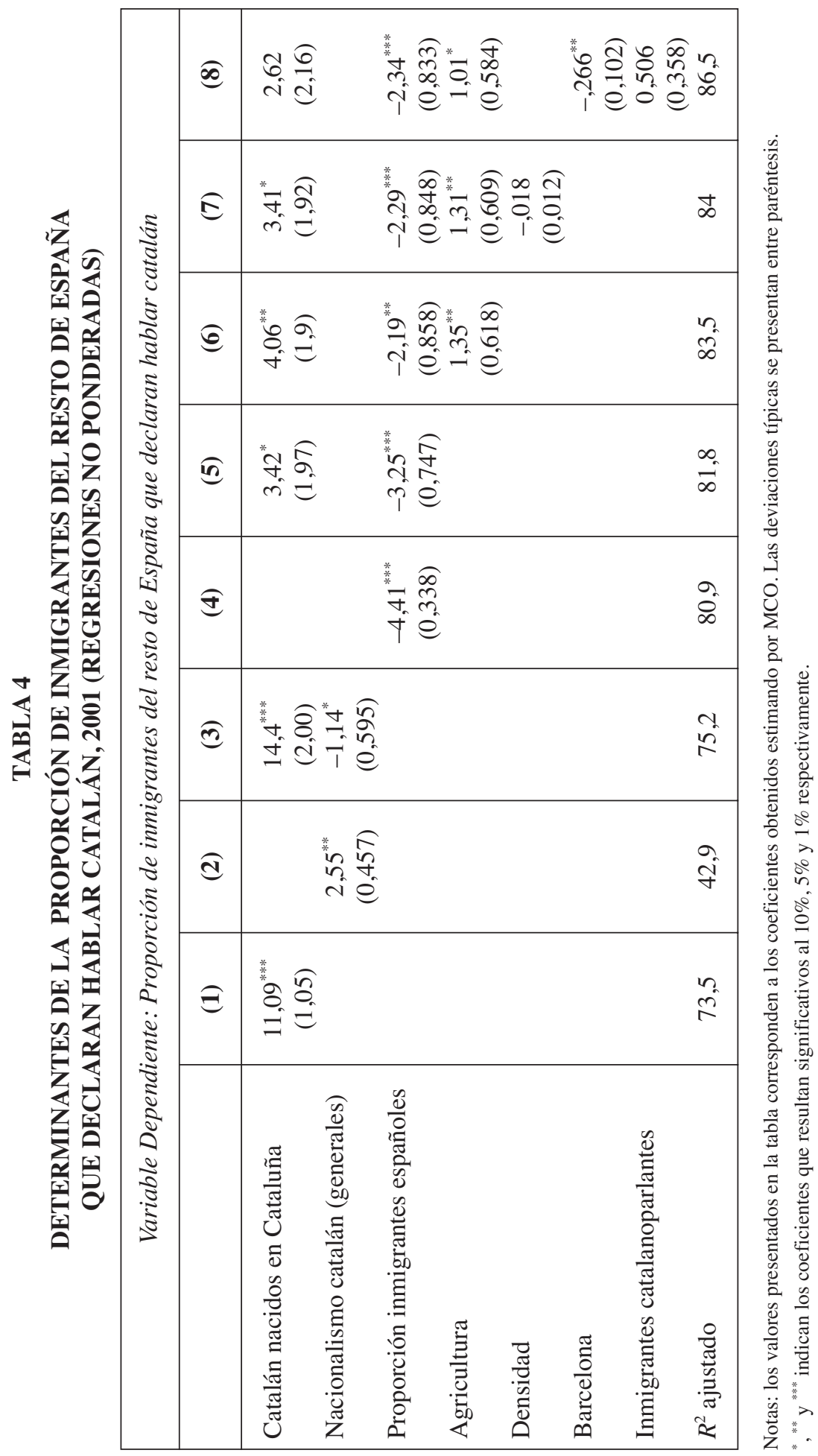




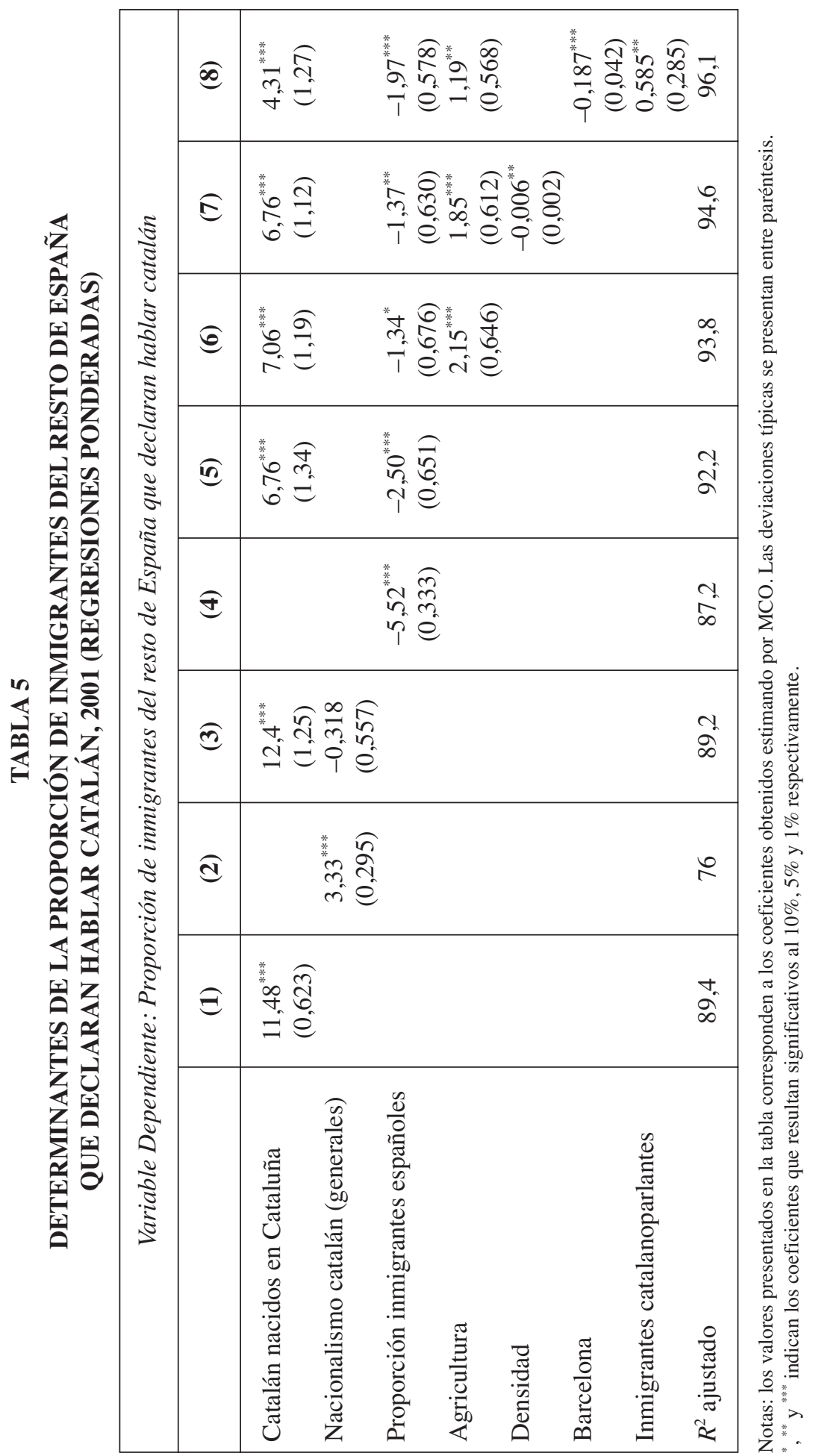


tranjero, mientras que la desagregación entre los nacidos en Cataluña y los nacidos en otras comunidades autónomas no está disponible. En principio, por lo tanto, podemos utilizar el nivel de educación de los inmigrantes como variable explicativa. Dado que la mayor parte del sistema de enseñanza en Cataluña tiene al catalán como lengua de instrucción, la utilización de esta variable está condicionada a la ausencia de un impacto del nivel de catalán de los inmigrantes extranjeros sobre su nivel de estudios, puesto que en ese caso la variable endógena explicaría a la variable exógena. Lo ideal sería disponer del nivel de educación de los inmigrantes extranjeros a su llegada a Cataluña, pero esa información no está disponible. Sin embargo, teniendo en cuenta que los datos sobre educación son de 2001, que cubren a la población extranjera de más de 10 años y que la llegada de los inmigrantes extranjeros se ha producido principalmente a partir de los años 90 , el porcentaje de extranjeros de más de 10 años escolarizados en 2001 en catalán debería ser relativamente bajo. La media del nivel de estudios de los extranjeros en cada comarca se hace atribuyendo un valor 0 a la proporción que «no sabe leer o escribir» o es «sin estudios», un valor igual a 1 para los que han obtenido un diploma de «primer grado», 2 para los que tienen un nivel de estudios «ESO, EGB, bachillerato elemental» $\mathrm{o}$ «FP medio», 3 para los que han acabado «FP superior» o un «bachillerato superior», 4 para los que han realizado una diplomatura, y 5 para los que han llegado al nivel «licenciatura o doctorado». Según esta definición, los extranjeros con un nivel medio de estudios más alto se sitúan en las comarcas del Garraf $(2,55)$, Barcelonès $(2,42)$ y Val d'Aran $(2,37)$, y los niveles de estudios más bajos en Osona $(1,2)$, Garroxta $(1,31)$ y Pla de l'Estany $(1,37)$.

Otra variable que podemos reconstruir (aunque sólo imperfectamente) a nivel comarcal para los inmigrantes extranjeros es la media del número de años desde su llegada a Cataluña. El sitio del IDESCAT ofrece datos anuales sobre el número de inmigrantes que se han instalado en cada comarca catalana entre 1988 y 2000, excepto para 1991 y 1996. Suponiendo que los individuos una vez instalados en una comarca permanecen en ella hasta 2001, podemos calcular para cada comarca el año medio de la llegada a Cataluña, y por lo tanto también la duración media de su estancia en Cataluña. Según estos datos, la comarca en la que los inmigrantes extranjeros se han instalado antes es el Baix Camp $(6,49$ años), seguida del Priorat $(6,27)$ y el Val d'Aran $(5,58)$. En el otro extremo, se sitúan el Plà de l'Estany $(2,11$ años de estancia media), la Cerdanya $(2,21)$ y el Solsonès $(2,39)$.

Finalmente, podemos también incluir más variables sobre el origen geográfico de los inmigrantes. En particular, incluimos una variable que indica la proporción comarcal de inmigrantes provinientes del continente americano («América») y del resto de la Unión Europea.

Los resultados de las regresiones se presentan en las tablas 6A y 6B. Presentamos sólo los resultados de las regresiones ponderadas, ya que los resultados de las regresiones no ponderadas son prácticamente idénticos. En la regresión (1), el nivel de estudios de los extranjeros tiene un impacto positivo y significativo sobre su conocimiento del catalán, y es éste un resultado que se repite en todas las regresiones salvo en la regresión (11). Su interpretación parece sencilla: aquellos inmigrantes con un nivel 
de estudios mayor tienen ceteris paribus menos dificultades para aprender una lengua adicional debido por ejemplo a su mayor familiariedad con las técnicas de aprendizaje. Al mismo tiempo, como en las regresiones para los otros grupos de inmigrantes, la densidad de población de la comarca tiene un impacto negativo.

Por su parte, el nivel de catalán de los residentes españoles en la comarca tiene un impacto positivo y significativo sobre el nivel de catalán de los inmigrantes extranjeros. Sin embargo, este resultado no se mantiene en otras regresiones. Más exactamente, el impacto de la variable «catalán de los residentes españoles» es positivo y significativo siempre que la regresión no incluya también como variable «nacionalismo catalán». A su vez, el nivel de voto a los partidos nacionalistas catalanes («nacionalismo catalán») tiene siempre un impacto positivo y significativo sobre el conocimiento del catalán de los inmigrantes extranjeros cuando no se considera en la misma regresión la variable «catalán residentes españoles». En los casos en los que las dos variables se incluyen «nacionalismo catalán» sigue siendo positiva y significativa en la mitad de las regresiones (es significativa en las regresiones (3), (9) y (15) y deja de serlo en las regresiones (6), (12) y (18)). Desde el punto de vista estadístico, la (frecuente) no significatividad de las dos variables cuando se consideran conjuntamente se explica por su fuerte correlación positiva $(0,82)$. Desde el punto de vista económico, si volvemos a nuestra interpretación de las secciones precedentes, y establecemos que la significatividad de la variable «catalán residentes españoles» reflejaría la importancia de los factores económicos en el aprendizaje del catalán, mientras que la significatividad del «nacionalismo catalán» reflejaría la importancia de la «presión ideológica», los resultados que obtenemos vendrían a indicar (i) la dificultad de distinguir entre los dos factores al estudiar el conocimiento del catalán por parte de los inmigrantes extranjeros (ii) un ligero predomio de los factores ideológicos, si nuestra interpretación es correcta.

La densidad de población tiene sistemáticamente un impacto negativo y significativo sobre el nivel de catalán de los inmigrantes extranjeros, y este resultado no parece estar ligado directamente al carácter agrícola de las comarcas o al hecho de que sean comarcas pirenaicas. Utilizamos por lo tanto la explicación propuesta en la sección 3: las comarcas con menor densidad estarían caracterizadas por una mayor utilización del catalán como lengua habitual, frente a una mayor utilización habitual del castellano en las comarcas más densamente pobladas, y esto explicaría que los inmigrantes extranjeros tengan un mejor conocimiento del catalán en las comarcas menos densamente pobladas.

La siguiente variable que consideramos es la proporción de inmigrantes extranjeros provinientes del continente americano. El coeficiente es positivo y significativo en la mitad de las regresiones consideradas, y no es significativo en el resto. Este resultado podría interpretarse en términos de proximidad lingüística: en aquellas comarcas con una mayor proporción de inmigrantes extranjeros procedentes de América, una mayor proporción de inmigrantes tiene como lengua materna una lengua cercana al catalán (en la inmensa mayoría de los casos, el castellano), y por lo tanto aprenden (más) fácilmente el catalán. Este resultado constituiría por lo tanto un elemento que indicaría que el desarrollo del conocimiento del catalán en los residentes 


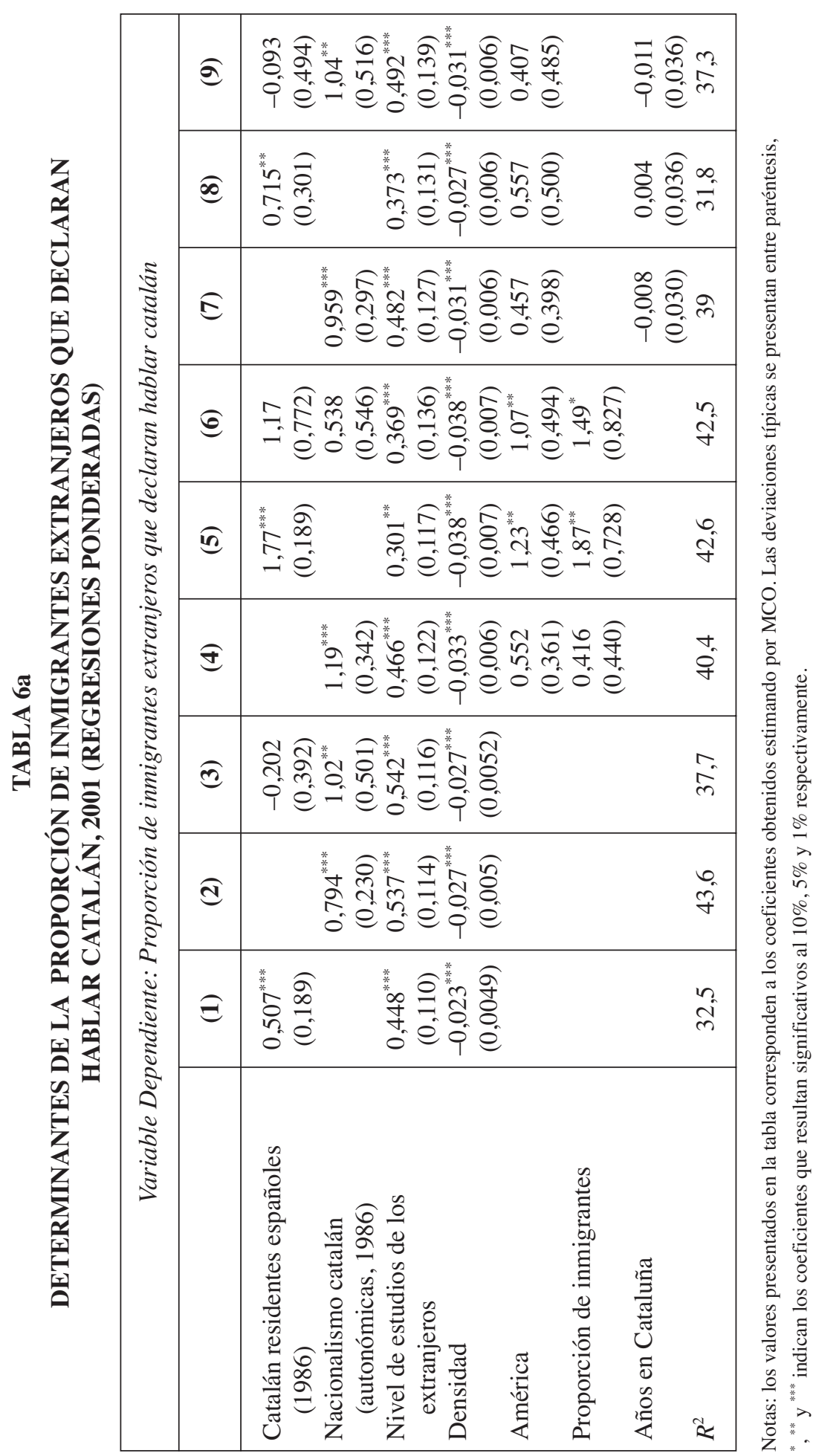


catalanes no está necesariamente obstaculizado por su conocimiento del castellano, sino que pueden existir en algunos casos complementariedades entre las dos lenguas.

En contraste con el resultado obtenido en la sección precedente, la proporción de inmigrantes (españoles y extranjeros) en la comarca tiene sorprendentemente un impacto positivo sobre el conocimiento del catalán de los inmigrantes extranjeros, aunque el coeficiente no es siempre significativo. Para entender este resultado, hay que tener en cuenta en primer lugar que el conjunto de los inmigrantes considerados en las regresiones de esta sección no tienen una lengua materna común, contrariamente a lo que ocurría (en una inmensa mayoría de los casos) cuando se consideraba sólo a los inmigrantes españoles, como en la sección precedente. Por lo tanto, de no aprender el castellano o el catalán, los inmigrantes extranjeros no castellanoparlantes podrán comunicarse con una proporción de individuos en la comarca muy inferior a la «proporción de inmigrantes» considerada en esta regresión. Esta hipótesis se confirma si se sustituye en las regresiones de las tablas 6A y 6B la «proporción de inmigrantes» por la «proporción de inmigrantes extranjeros», ya que esta nueva variable no tiene nunca un impacto significativo sobre el conocimiento del catalán de los inmigrantes extranjeros. Para entender por qué el efecto es positivo y significativo en algunos casos, pueden tenerse en cuenta los elementos siguientes: (i) la proporción de inmigrantes extranjeros en el total de inmigrantes está correlacionada negativamente con la proporción de inmigrantes en la comarca (ii) el nivel de catalán de los inmigrantes del resto de España es mayor en 2001 que el nivel de catalán de los inmigrantes extranjeros. Por lo tanto, en aquellas comarcas con una alta proporción de inmigrantes, los inmigrantes extranjeros convivirán con inmigrantes que en términos medios podrán comunicar en catalán, lo cual aumentará sus propios incentivos a aprender catalán.

En la tabla 6A, el número de años pasados en Cataluña no tiene un impacto significativo sobre la proporción de inmigrantes extranjeros que declaran hablar catalán. Para un grupo de inmigrantes con una lengua materna dada, esperamos que una estancia más larga en Cataluña tenga un impacto positivo sobre su nivel de catalán. Sin embargo, en nuestras regresiones, los inmigrantes extranjeros tienen diferentes lenguas maternas, y por lo tanto deben realizar esfuerzos diferentes para aprender el catalán, y además no llegaron a Cataluña necesariamente en el mismo momento. Por lo tanto, debido a este efecto de composición, no existe ninguna razón por la que el número de años en Cataluña deba necesariamente estar correlacionado positivamente con la variable endógena.

Como indica la tabla 6B, la proporción de inmigrantes provinientes del resto de la Unión Europea tiene a veces un impacto positivo sobre la variable endógena. Este resultado podría ser debido al mayor nivel de estudios de los inmigrantes europeos, como indica el hecho de que la regresión en la que esta variable es significativa (la regresión (11)) es la única en la que el «nivel de estudios de los extranjeros» no tiene un impacto significativo sobre la variable endógena.

Por último, como en las regresiones de la sección 3, la variable indicativa «Pirineos» tiene un impacto positivo sobre el conocimiento del catalán por parte de los inmigrantes. 


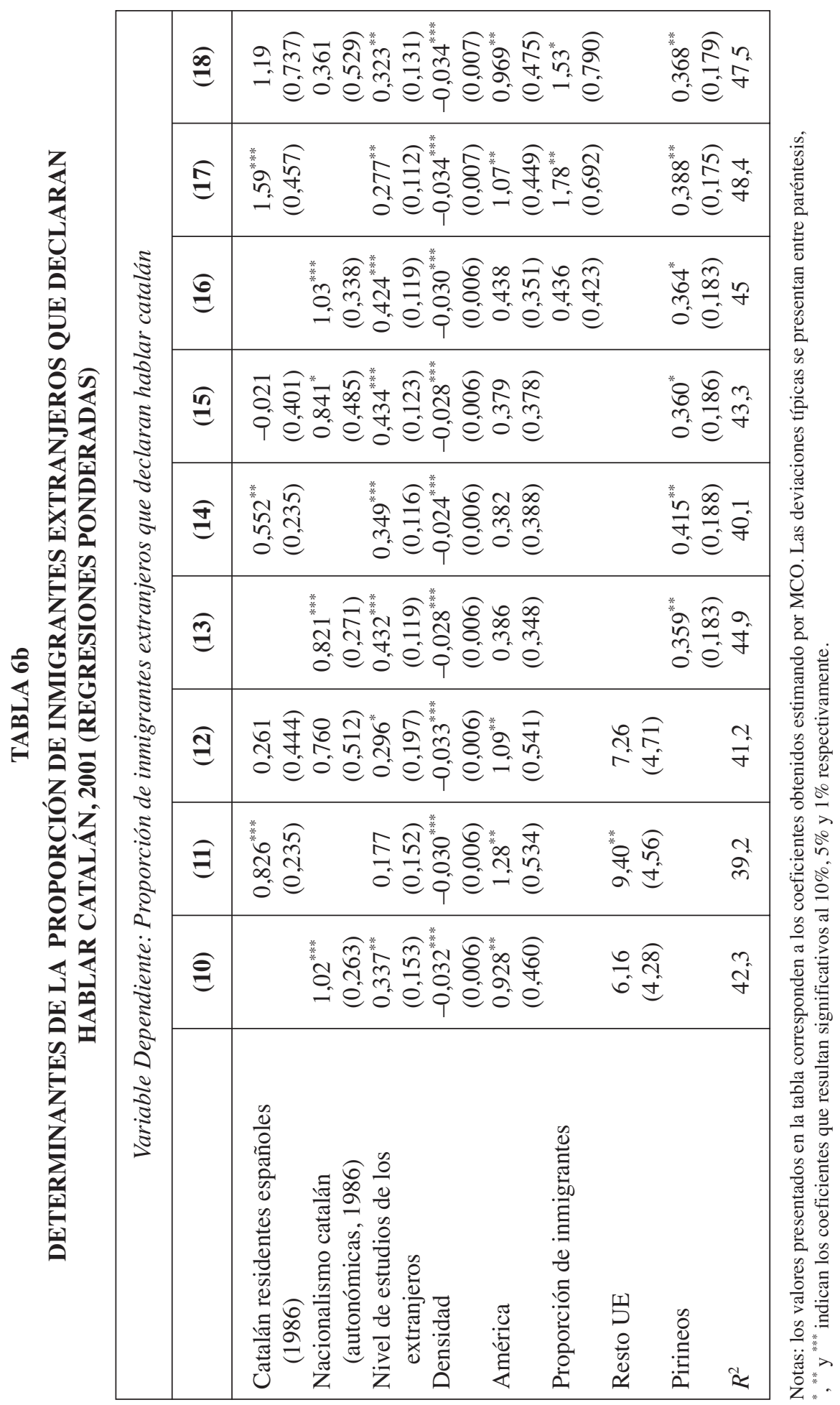




\section{Conclusión}

Este artículo muestra que los factores económicos pueden ser importantes para determinar el aprendizaje de una lengua por parte de los inmigrantes. Para el caso de Cataluña, mostramos que la proporción de catalanoparlantes entre los nacidos en Cataluña tiene un impacto positivo sobre el conocimiento del catalán de los inmigrantes en su conjunto, y que los inmigrantes nacidos en el resto de España hablan más catalán en aquellas comarcas en las que la proporción de inmigrantes españoles es menor. Sin embargo, los resultados obtenidos para los inmigrantes extranjeros muestran que los factores ideológicos también pueden ser relevantes.

Los resultados diferentes obtenidos para los inmigrantes nacidos dentro y fuera de España podrían deberse a la transformación política de Cataluña entre los años 50 y 90, a la fragmentación lingüística de la inmigración extranjera (frente a la homogeneidad lingüística de la inmigración española) o simplemente al hecho de que la inmigración extranjera es mucho más reciente.

Algunos de nuestros resultados indican que pueden existir ciertas «complementariedades» entre el castellano y el catalán en Cataluña, en particular porque el nivel de conocimiento del catalán es mayor en aquellas comarcas con una fuerte proporción de inmigrantes castellanoparlantes (españoles o extranjeros).

Por último, nuestro análisis indica que la variable «densidad» tiene un fuerte impacto negativo sobre el conocimiento del catalán de los inmigrantes nacidos fuera de España, resultado que atribuimos al hecho de que el castellano sea (relativamente) más utilizado en las zonas más densamente pobladas. Este tipo de resultados indica que la falta de datos sobre el conocimiento del castellano en Cataluña limita de forma muy importante nuestra capacidad de comprensión de la integración lingüística de los inmigrantes extranjeros.

\section{Referencias bibliograficas}

[1] CAPELLADES, J. (2001) «Los censos lingüísticos en Cataluña 1975-2001», Revista Fuentes Estadísticas, INE, Julio-Agosto-Septiembre.

[2] CHURCH, J. y I. KING (1993) «Bilingualism and network externalities» Canadian Journal of Economics-Revue Canadienne d'Economique, XXVI No. 2.

[3] IDESCAT (2003) Estadística d'Usos Lingüístics a Catalunya, 2003.

[4] LAZEAR, E. (1999) «Culture and Language» Journal of Political Economy 107 (6), S95-126.

[5] ORTEGA, J. y G. VERDUGO (2006) «Immigrant Assimilation in a Bilingual Country». Mimeo.

[6] RENDON, S. (2006) «The Catalan premium: language and employment in Catalonia», Journal of Population Economics (de próxima publicación). 


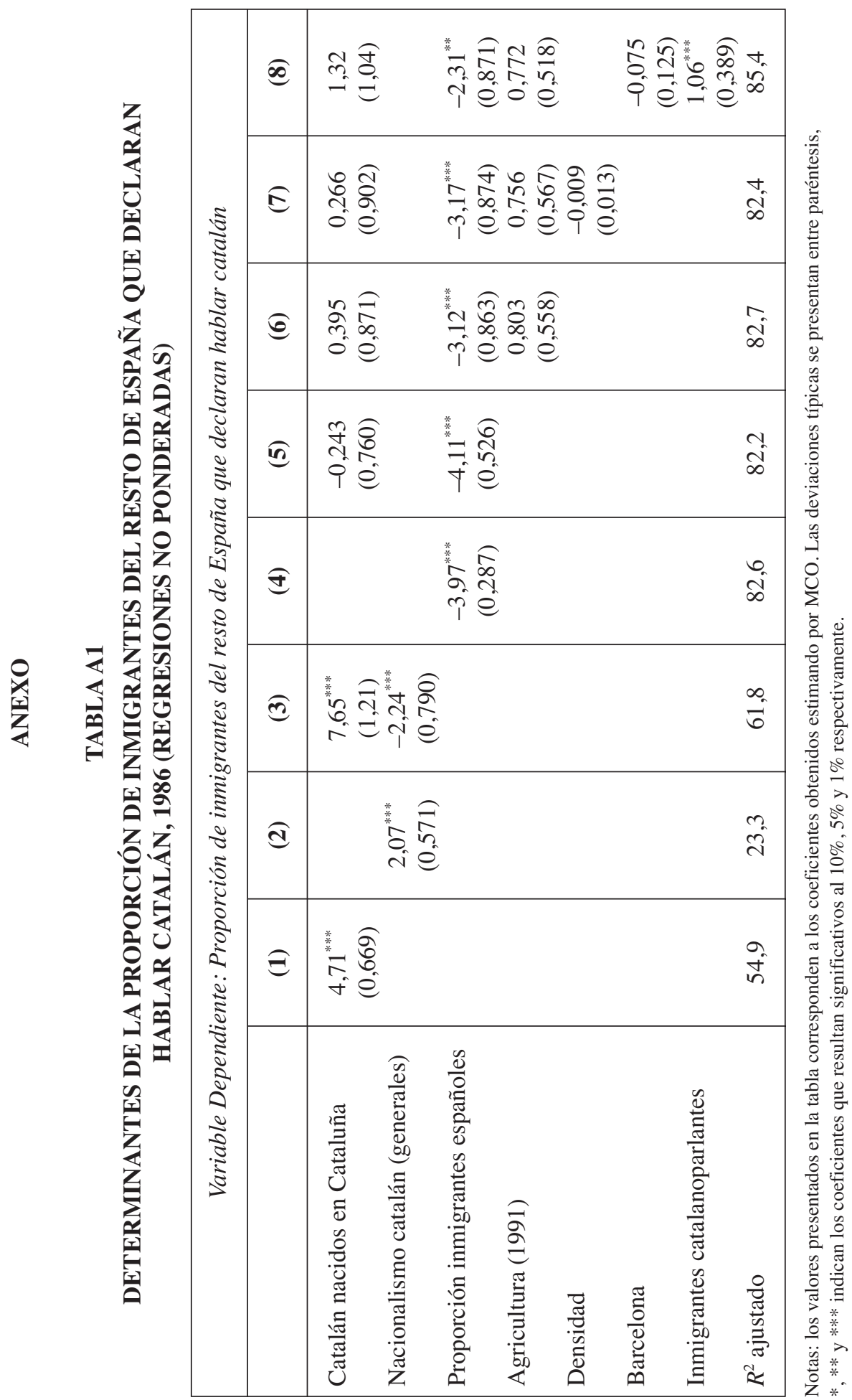




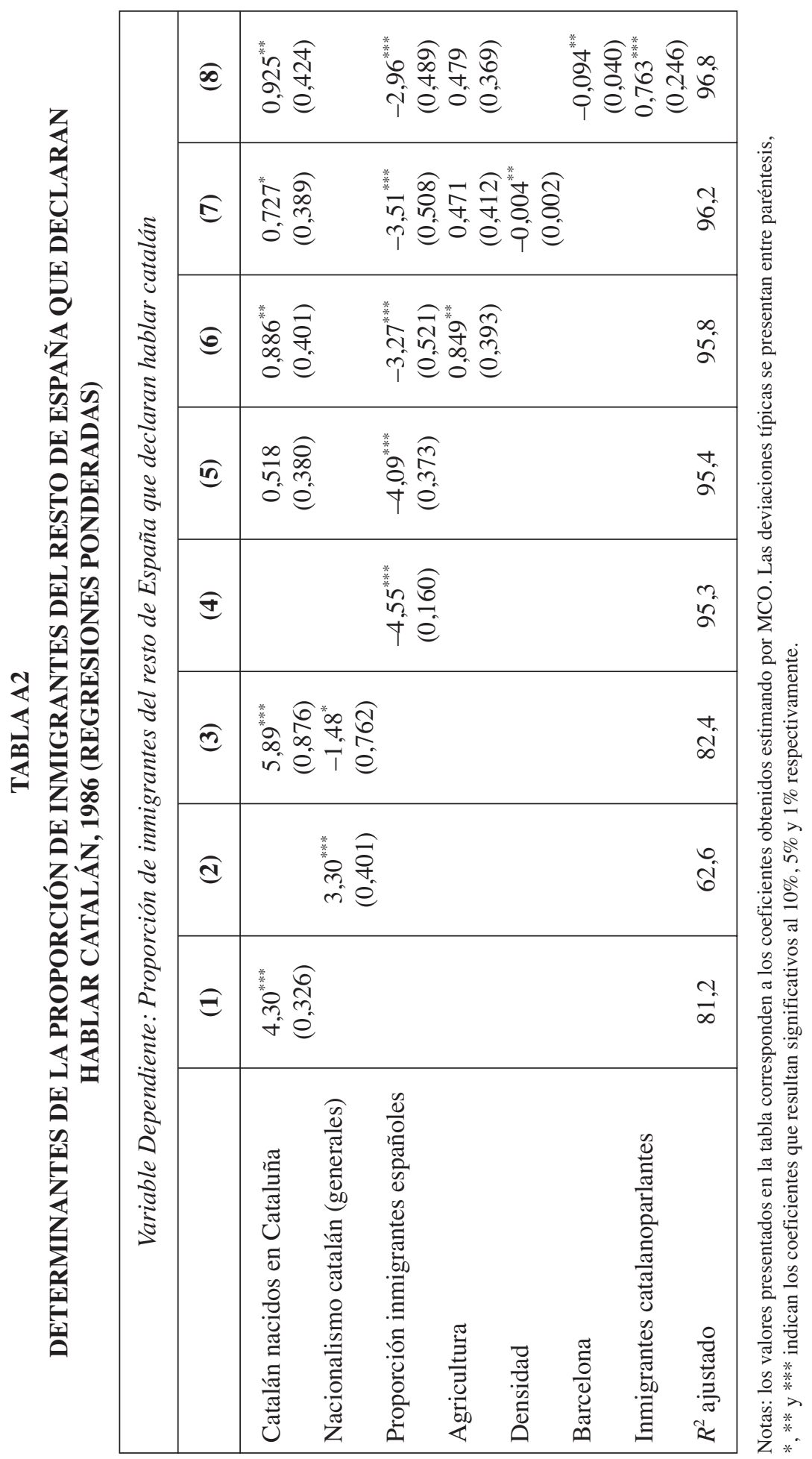


